\title{
Quantitative chest CT analysis in COVID-19 to predict the need for oxygenation support and intubation
}

\author{
Ezio Lanza ${ }^{1}$ (1) - Riccardo Muglia ${ }^{2}$ - Isabella Bolengo ${ }^{2}$ - Orazio Giuseppe Santonocito ${ }^{2} \cdot$ Costanza Lisi $^{3}$. \\ Giovanni Angelotti ${ }^{4} \cdot$ Pierandrea Morandini $^{4} \cdot$ Victor Savevski $^{4} \cdot$ Letterio Salvatore Politi $^{1,3} \cdot$ Luca Balzarini $^{1}$
}

Received: 14 April 2020 / Revised: 20 May 2020 / Accepted: 5 June 2020 / Published online: 26 June 2020

(C) European Society of Radiology 2020

\begin{abstract}
Objective Lombardy (Italy) was the epicentre of the COVID-19 pandemic in March 2020. The healthcare system suffered from a shortage of ICU beds and oxygenation support devices. In our Institution, most patients received chest CT at admission, only interpreted visually. Given the proven value of quantitative CT analysis (QCT) in the setting of ARDS, we tested QCT as an outcome predictor for COVID-19.

Methods We performed a single-centre retrospective study on COVID-19 patients hospitalised from January 25, 2020, to April 28, 2020, who received CT at admission prompted by respiratory symptoms such as dyspnea or desaturation. QCT was performed using a semi-automated method (3D Slicer). Lungs were divided by Hounsfield unit intervals. Compromised lung $(\% \mathrm{CL})$ volume was the sum of poorly and non-aerated volumes $(-500,100 \mathrm{HU})$. We collected patient's clinical data including oxygenation support throughout hospitalisation.

Results Two hundred twenty-two patients (163 males, median age 66, IQR 54-6) were included; 75\% received oxygenation support (20\% intubation rate). Compromised lung volume was the most accurate outcome predictor (logistic regression, $p<0.001) . \% \mathrm{CL}$ values in the 6-23\% range increased risk of oxygenation support; values above $23 \%$ were at risk for intubation. $\% \mathrm{CL}$ showed a negative correlation with $\mathrm{PaO}_{2} / \mathrm{FiO}_{2}$ ratio $(p<0.001)$ and was a risk factor for in-hospital mortality $(p<0.001)$. Conclusions QCT provides new metrics of COVID-19. The compromised lung volume is accurate in predicting the need for oxygenation support and intubation and is a significant risk factor for in-hospital death. QCT may serve as a tool for the triaging process of COVID-19.

Key Points

- Quantitative computer-aided analysis of chest CT (QCT) provides new metrics of COVID-19.

- The compromised lung volume measured in the - 500, $100 \mathrm{HU}$ interval predicts oxygenation support and intubation and is a risk factor for in-hospital death.

- Compromised lung values in the 6-23\% range prompt oxygenation therapy; values above $23 \%$ increase the need for intubation.
\end{abstract}

Keywords COVID-19 $\cdot$ Tomography, spiral computed $\cdot$ Intubation $\cdot$ Pulmonary ventilation

Ezio Lanza

eziolanza@gmail.com

1 Department of Diagnostic and Interventional Radiology, Humanitas Clinical and Research Center - IRCCS, Via A. Manzoni 56, Rozzano, 20089 Milan, Italy

2 Training School in Radiology, Humanitas University, Via Rita Levi Montalcini 4, Pieve Emanuele, 20090 Milan, Italy

3 Department of Biomedical Sciences, Humanitas University, Via Rita Levi Montalcini 4, Pieve Emanuele, 20090 Milan, Italy

4 Artificial Intelligence Center, Humanitas Clinical and Research Center - IRCCS, Via A. Manzoni 56, Rozzano, 20089 Milan, Italy

$\begin{array}{ll}\text { Abbreviations } \\ \text { \%CL } & \text { Compromised lung } \\ \text { \%NAL } & \text { Normally aerated lung } \\ \text { \%NNL } & \text { Non-aerated lung } \\ \text { \%PAL } & \text { Poorly aerated lung } \\ \text { ARDS } & \text { Acute Respiratory Distress Syndrome } \\ \text { BMI } & \text { Body mass index } \\ \text { COVID-19 } & \text { Coronavirus disease 2019 } \\ \text { CT } & \text { Computed tomography } \\ \text { HU } & \text { Hounsfield units } \\ \text { IQR } & \text { Interquartile range } \\ \text { QCT } & \text { Quantitative computed tomography }\end{array}$


rtRT-PCR Real-time reverse transcriptase-polymerase chain reaction

\section{Introduction}

In the early days of December 2019, the first pneumonia cases of a new coronavirus named SARS-CoV-2 were identified in Wuhan, the capital city of Hubei province (China) [1]. As of April 30, 2020, 1,112,667 cases have been reported in the EU/ EEA and the UK: Spain $(212,917)$ and Italy $(203,591)$ had the largest contagion [2].

Since the initial spread of this new illness, known as coronavirus disease 2019 (COVID-19), many patients have been hospitalized with respiratory symptoms [3]. The clinical spectrum is broad, including asymptomatic infection, mild upper respiratory tract disease and severe interstitial pneumonia with respiratory failure requiring oxygenation support or intubation $[4,5]$.

Computed tomography (CT) is the most sensitive radiological technique for the diagnosis of COVID-19, showing diffuse lung alterations ranging from ground-glass opacities to parenchymal consolidations; several radiological patterns are observed at different times throughout the disease course [6, 7]. However, CT has limited specificity for distinguishing between virus [8]. Early diagnosis is hence mainly performed with nasopharyngeal swabs and virus RNA extraction with real-time reverse transcriptase-polymerase chain reaction (rtRT-PCR) [9].

Recently, the visual assessment of lung damage on CT scans has been proven valuable in determining the prognostic implications [10]. Nonetheless, computer-aided quantitative analysis of the CT exam (Quantitative Computed Tomography [QCT]) can also be used for this purpose, as already demonstrated by the research on the acute respiratory distress syndrome (ARDS) [11, 12].

The main objective of our research was to explore the role of QCT analysis as an outcome predictor for COVID-19. To this end, we conducted a monocentric retrospective study on positive patients in a tertiary referral Institution in Milan (Lombardy, Italy) during the recent outbreak, where we experienced hospital overcrowding and shortage of intensive care unit (ICU) beds, mechanical ventilation devices and oxygen [13].

\section{Materials and methods}

\section{Study oversight}

The present is an observational retrospective study, conducted in a tertiary referral University Hospital in Milan (Lombardy, Italy). The study protocol followed the ethical guidelines established in the 1975 Declaration of Helsinki, compliant with the procedures of the local ethical committee, and was approved by the Institutional Review Board. This study received no financial support.

\section{Data sources}

We obtained laboratory, clinical and radiological data of hospitalised patients affected by COVID-19 from the electronic medical records; the inclusion data cutoff for the analyses was January 25, 2020, and April 28, 2020.

COVID-19 was diagnosed based on a positive result on rtRT-PCR assay on nasal and pharyngeal swab specimens [14]. We included only laboratory-confirmed cases who received a non-contrast chest CT at admittance in the emergency department.

We analysed patients' demographics and clinical and laboratory findings at admittance from electronic medical records. Patient demographics included age, sex, body mass index (BMI), concomitant/previous diseases and smoking habit. Clinical and laboratory assessments consisted of body temperature, $\mathrm{PaO}_{2}, \mathrm{PaCO}_{2}$, and $\mathrm{C}$-reactive protein. We also noted the time from symptom onset, days of hospitalisation, ICU admittance, medical therapy administered and the most invasive level of oxygenation support provided. In particular, we distinguished between low-flow oxygenation (nasal cannula, face mask), high-flow oxygenation (Venturi mask, helmet CPAP) and mechanical ventilation through an endotracheal tube. We collected the time interval from CT and oxygenation support, as well as the first $\mathrm{PaO}_{2} / \mathrm{FiO}_{2}$ ratio available. In-hospital deaths and healed patients' discharge dates were also noted.

The clinical features of confusion (mental test score of 8 or less), urea, respiratory rate and blood pressure were also acquired to calculate the CURB-65, a validated score to predict the severity of community-acquired pneumonia [15] that stratifies patients in groups from 1 to 3 according to the risk of mortality.

\section{Chest CT and quantitative analysis}

All patients received a standard non-contrast chest $\mathrm{CT}$ with a multidetector CT scanner (Philips Brilliance) with the following setup: collimation, $64 \times 0.25$; voltage, $120 \mathrm{kV}$; tube current, 130-200 mAs, $240 \mathrm{~mA}$, pitch 1.4, slice thickness after reformat, $2.5 \mathrm{~mm}$. The field of view included the whole chest and was acquired during forced inspiration, in keeping with patient compliance. The dataset was anonymised and exported to a dedicated segmentation suite for medical image computing (3D Slicer, www.slicer.org) [16] equipped with a semiautomated segmentation algorithm (Chest Imaging Platform) [17]. This software, validated as useful in the surgical setting [18], performed a first-pass automated segmentation; then, lung volumes were manually perfected using threedimensional tools such as spherical brushes or erasers.

As a rule, a complete segmentation included both lungs with interstitial structures, segmentary vessels and bronchi; the main pulmonary arteries and bronchi, all mediastinal 
structures and eventual pleural effusion were excluded, as well as lung masses (e.g. tumours, fungal disease).

Lung volumes, considered percentages of the total volume, were extracted according to different Hounsfield unit (HU) intervals into non-aerated (\%NNL, density between 100, $100 \mathrm{HU})$, poorly aerated (\%PAL, $-101,-500 \mathrm{HU})$, normally aerated (\%NAL, $-501,-900 \mathrm{HU})$, and hyperinflated (-901, $-1000 \mathrm{HU})$ [19]. The additional volume "compromised lung" $(\% \mathrm{CL})$ was considered the sum of $\% \mathrm{PAL}$ and $\% \mathrm{NNL}(-500$, $100 \mathrm{HU}$ ) (Fig. 1). The authors in charge of segmentation (E.L., C.L., R.M.) were unaware of the laboratory and clinical parameters or hospitalisation outcomes. Conflicts were resolved in consensus. The principal investigator reviewed and confirmed all segmentations before data entry. The time needed to complete each analysis was recorded.

\section{Outcomes}

The primary objective was to identify and validate the most accurate lung volume derived by QCT, to predict the two main study outcomes: the need for oxygenation support and the need for intubation in patients affected by COVID-19.

Other objectives included correlation with pulmonary dysfunction as measured by the $\mathrm{PaO}_{2} / \mathrm{FiO}_{2}$ ratio and prediction of in-hospital death (Table 1).

\section{Statistical analysis}

\section{Development of prediction models}

All analyses were performed using Stata 13 (StataCorp LP). Multiple binomial logistic regressions were performed to explore the correlation of the lung volumes, \%NAL, \% PAL and \%CL, over the two outcomes of interest. All clinically relevant predictors without missing data were included in the final model as covariates: age, sex, smoke habit, CPR, heart disease, chronic lung disease, cancer, diabetes, chronic kidney failure, urea levels and CURB-65 group. Three similar models were thus developed: \%NAL-model, \%PAL-model and \%CL-model.
BMI was available for 161 patients and was tested in a separate model.

A Pearson's product-moment correlation was run to assess the relationship between the selected lung interval and $\mathrm{PaO}_{2} /$ $\mathrm{FiO}_{2}$ in 106 patients (nasal cannula $=26$, Venturi mask $=28$, helmet $\mathrm{CPAP}=21$, endotracheal tube $=27$ ).

A Cox regression survival analysis was performed to explore potential predictors of mortality. All potential candidates were tested with univariate analyses; the cutoff for inclusion in the final model was set at $p<0.2$.

\section{Model validation}

All simulations were run using Python programming language (Python Software Foundation, https://www.python.org/). Categorical variables were preliminarily tested for correlation using chi-square tests; Wilcoxon rank-sum tests were performed on continuous covariates to inspect the probability of being sampled from the same distribution. Two separate multivariate regressions, without regularisation, were performed on both outcomes over the space of covariates. Models' coefficients, confidence intervals and their associated associations ( $p$ values) were investigated to assess whether the selected lung interval remained significant despite adjusting for possible confounders. Predictive machine learning models were built using logistic regression with regularisation. To adjust for class imbalance, on both outcomes, and preserve the limited amount of available observations, the logistic regression was stacked upon a SMOTE model during training [20].

Hyperparameters were chosen by randomised selection over 1000 possible validations by means of 10 -fold cross-validation each, for a total of 5000 actually trained models. The aim was to alleviate class imbalance by maximisation of class weighted F1 score, harmonic mean of precision and recall (Fig. 2).

Cross-validated receiver operating characteristic (CVROC) and mean areas under the curve (CV-AUC) were calculated [21]. Different cross-validated cut points at $90 \%$ sensitivity and at $90 \%$ specificity were estimated for both outcomes.

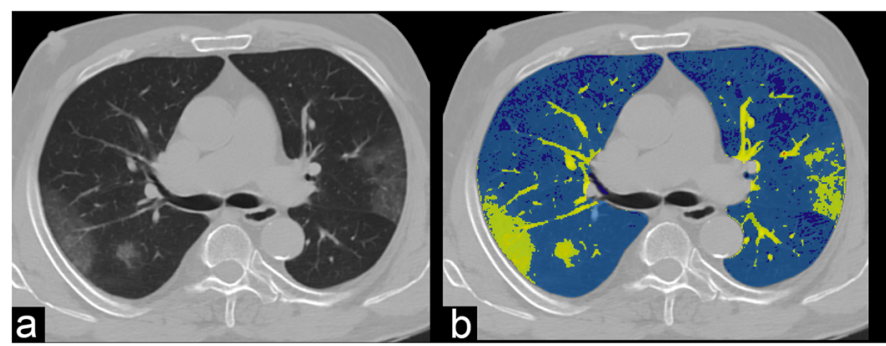

Fig. 1 Quantitative lung CT analysis of an 81-year-old male patient affected by COVID-19. a Non-contrast chest CT at admission showing bilateral ground-glass opacities, common findings of the novel coronavirus pneumonia. b Semi-automated segmentation using 3D Slicer. Blue areas are normal lung parenchyma; yellow areas represent poorly aerated

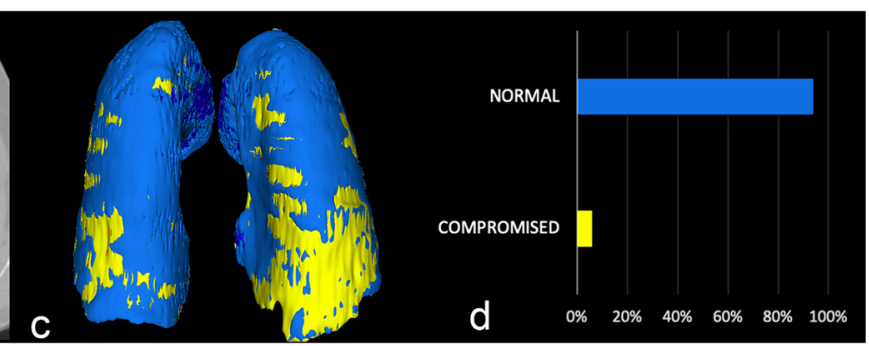

lung in the $-500,-100 \mathrm{HU}$ interval. c $3 \mathrm{D}$ volumetric representation of both lungs. d Comparison between normal and compromised lung volumes. This patient had $6 \%$ of compromised lung volume, required no oxygenation support and was discharged after 15 days of observation and supportive therapy 
Table 1 Multivariate analyses of risk factors for oxygenation support, intubation and in-hospital death

\begin{tabular}{|c|c|c|c|c|c|c|c|c|c|c|c|c|}
\hline \multirow{3}{*}{$\frac{\text { Risk factor }}{\text { Compromised lung volume }}$} & \multicolumn{4}{|c|}{ Oxygenation support } & \multicolumn{4}{|c|}{ Intubation } & \multicolumn{4}{|c|}{ Death } \\
\hline & \multirow{2}{*}{$\frac{\mathrm{OR}}{1.28}$} & \multicolumn{2}{|c|}{$95 \% \mathrm{CI}$} & \multirow{2}{*}{$\frac{p \text { value }}{<0.001 *}$} & \multirow{2}{*}{$\frac{\mathrm{OR}}{1.12}$} & \multicolumn{2}{|c|}{$95 \% \mathrm{CI}$} & \multirow{2}{*}{$\frac{p \text { value }}{<0.001 *}$} & \multirow{2}{*}{$\frac{\mathrm{OR}}{1.03}$} & \multirow{2}{*}{$\frac{p \text { value }}{0.001 *}$} & \multicolumn{2}{|c|}{$95 \% \mathrm{CI}$} \\
\hline & & 1.16 & 1.41 & & & 1.07 & 1.17 & & & & 1.01 & 1.04 \\
\hline Age & 1.04 & 1.00 & 1.08 & 0.052 & 0.97 & 0.92 & 1.01 & 0.154 & 1.07 & $<0.001 *$ & 1.03 & 1.10 \\
\hline Sex & 1.59 & 0.63 & 4.04 & 0.329 & 1.04 & 0.33 & 3.24 & 0.952 & 0.93 & 0.836 & 0.47 & 1.83 \\
\hline Smoke habit & 1.03 & 0.17 & 6.12 & 0.974 & 1.08 & 0.15 & 7.57 & 0.939 & 1.77 & 0.129 & 0.85 & 3.68 \\
\hline CRP & 1.02 & 0.98 & 1.05 & 0.321 & 1.01 & 0.98 & 1.05 & 0.506 & 1.03 & $0.001 *$ & 1.01 & 1.05 \\
\hline Heart disease & 2.23 & 0.45 & 11.05 & 0.326 & 0.18 & 0.02 & 1.79 & 0.143 & 1.19 & 0.642 & 0.58 & 2.44 \\
\hline Lung disease & 6.87 & 0.70 & 67.95 & 0.099 & 0.65 & 0.07 & 6.14 & 0.703 & 1.05 & 0.904 & 0.47 & 2.33 \\
\hline Cancer & 0.87 & 0.21 & 3.64 & 0.848 & 0.73 & 0.11 & 4.71 & 0.739 & 3.42 & $0.003 *$ & 1.52 & 7.73 \\
\hline Diabetes & 0.88 & 0.31 & 2.52 & 0.809 & 0.87 & 0.23 & 3.23 & 0.833 & 1.88 & 0.052 & 0.99 & 3.55 \\
\hline CKD & 1.53 & 0.13 & 18.18 & 0.738 & 0.69 & 0.07 & 7.01 & 0.752 & 4.14 & $0.003 *$ & 1.60 & 10.70 \\
\hline CURB- $65^{\mathrm{a}} 1$ & 0.58 & 0.06 & 5.25 & 0.630 & 1.31 & 0.18 & 9.40 & 0.788 & 0.81 & 0.685 & 0.30 & 2.23 \\
\hline CURB-65 2 & 0.32 & 0.05 & 2.04 & 0.228 & 1.80 & 0.40 & 8.09 & 0.443 & 0.80 & 0.556 & 0.38 & 1.67 \\
\hline Urea at admission & 1.60 & 0.49 & 5.21 & 0.437 & 4.93 & 1.39 & 17.47 & $0.014^{*}$ & 1.71 & 0.179 & 0.78 & 3.75 \\
\hline BMI $(n=161)$ & 1.08 & 0.98 & 1.20 & 0.130 & 0.95 & 0.84 & 1.08 & 0.428 & .. & .. & .. & .. \\
\hline
\end{tabular}

$O R=$ odds ratio $C I=$ confidence interval; $C R P=\mathrm{C}$-reactive protein; $C K D=$ chronic kidney disease. ${ }^{\text {a }}$ Risk group according to the $\mathrm{CURB}-65$ scoring system

* italics mark statistical significance

\section{Results}

\section{Demographic and clinical characteristics}

A cohort of 222 patients (163 males, 59 females, median age 66, interquartile range [IQR] 54-6) was identified (Table 2). The median interval time between symptom onset at admission and CT was 7 days (IQR, 3-8). The median body temperature during the $\mathrm{CT}$ exam was $37.7^{\circ} \mathrm{C}$ (IQR, 36.9 $-38.4^{\circ}$ ), and the median $\mathrm{PaO}_{2}$ was $67 \mathrm{mmHg}$ (IQR, 57-90). The median hospitalisation length was 11 days (IQR, 7-17, maximum 56).

During the hospital stay, $75 \%$ of patients required oxygenation support (Table 3) as follows: $29 \%$ low-flow oxygenation (26\% nasal cannula, $3 \%$ facial mask); $26 \%$ high-flow oxygenation (16\% Venturi mask, 10\% helmet CPAP); and 20\% mechanical ventilation with an endotracheal tube. Median $\mathrm{PaO}_{2} /$ $\mathrm{FiO}_{2}$ ratio, as recorded on the first day of oxygenation support, was 192.0 (IQR, 122.2-251.5). The median time interval from admission CT to oxygenation support was 1 day (IQR, 0-2).

During the observation, antiviral therapy with lopinavirritonavir was administered in $55 \%$ of patients and darunavir plus cobicistat in $36 \%$. Hydroxychloroquine was used in $91 \%$. All patients also received the best medical therapy tailored to the individual case, including broad-spectrum antibiotics.

Up to the reference date April 28, 2020, out of total 222 patients, $8(3.6 \%)$ were still hospitalised, whereas the remaining $214(96.3 \%)$ were discharged, $150(67.6 \%)$ of them healed and 64 (28.8\%) died after a median 9 days hospitalisation (IQR, 5-14): their median age was 75.7 years (IQR, 71-80).

Regarding the CURB-65 score, 129 patients were categorised as low risk (group 1), and 57 patients were medium risk (group 2); no patients were scored as high risk (group 3). Death rates were $19 \%$ and $36 \%$ respectively.

\section{Diagnostic performance of the different lung volumes}

All CT scans were considered of diagnostic quality and successfully analysed. All lung volumes were correctly extracted (Table 3). The median time for segmentation was $11 \mathrm{~min}$ (IQR, 7-14).

The need for oxygenation support was accurately predicted both by the \%PAL model (LR $\left.\chi^{2(13)}, 86.05 ; p<0.0001\right)$ and by the $\%$ CL model (LR $\left.\chi^{2(13)}, 86.90 ; p<0.0001\right)$. In both, \%PAL and $\%$ CL showed a strong predictive value $(p<0.0001)$ and were the only significant covariates. The $\%$ NAL model showed worse performance $\left(\mathrm{LR} \chi^{2(13)}, 39.18 ; p<0.0002\right)$ and \%NAL was not a significant predictor $(p=0.315)$.

The need for intubation was accurately predicted by all three models with minimal differences in LR $\chi^{2(13)}$ (\% NAL model, 81.47; \%PAL model, 85.31, \%CL model, 89.88; $p<0.0001$ each) and all three lung volumes showed strong predictive value $(p<0.0001)$. The compromised lung volume was considered the best single predictor of both outcomes, and the $\% \mathrm{CL}$ model was chosen for validation.

A separate model was fitted adding BMI as a covariate in a subgroup of 161 patients, proving no effect on both outcomes (oxygenation support, $p=0.130$; intubation, $p=0.428$ ).

\section{Validation of compromised lung volume}

For the outcome of oxygenation support, the \%CL model reached $\mathrm{CV}$-AUROC 0.83 (weighted f1-score, 0.72; standard deviation [SD], 0.098). Cross-validated cut points were 
Compromised lung volume as a predictor of oxygenation support
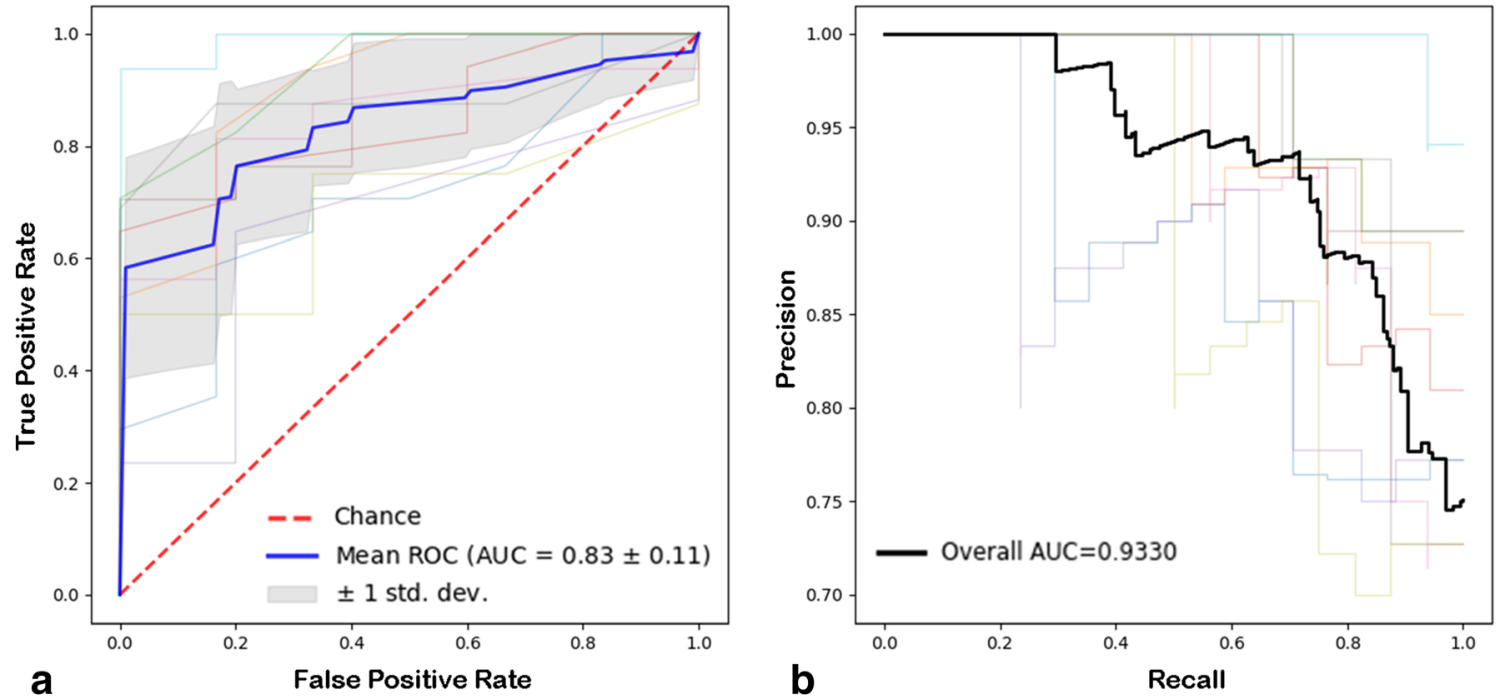

\section{Compromised lung volume as a predictor of intubation}
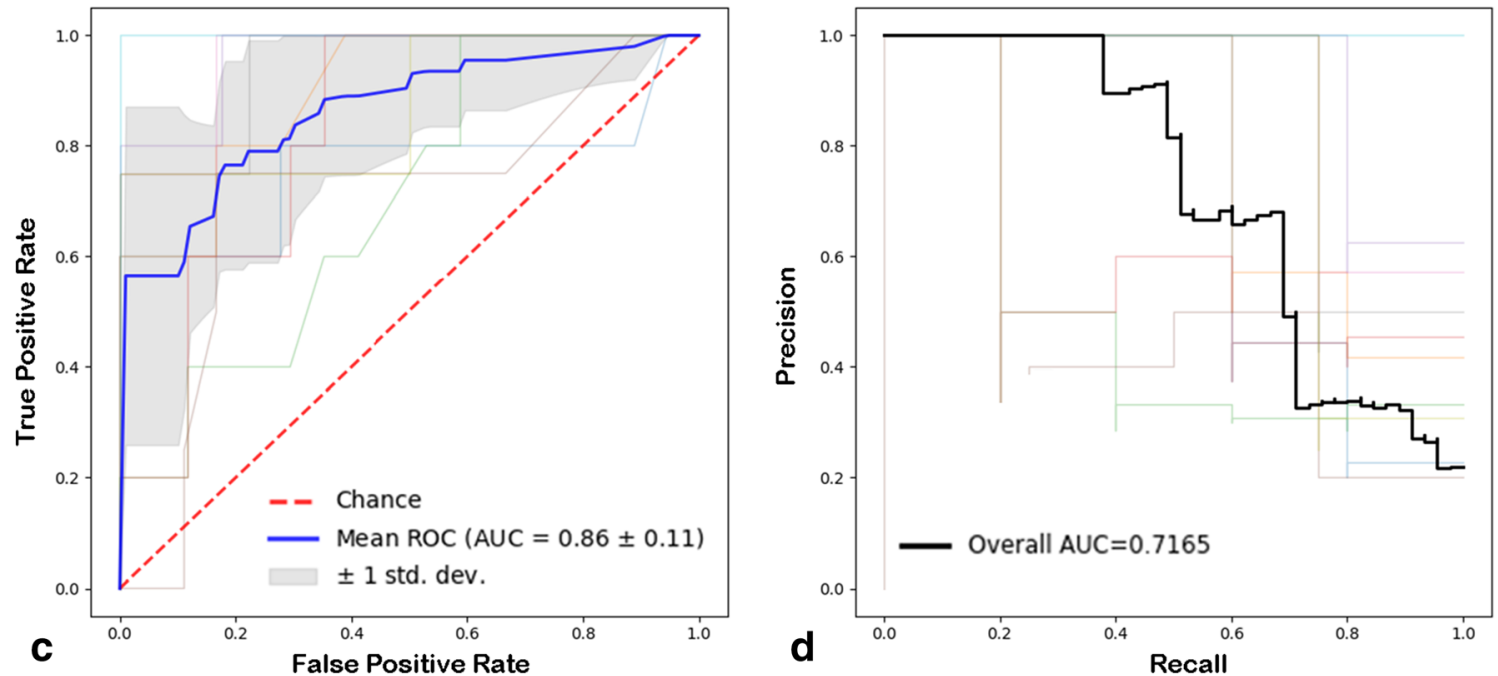

Fig. 2 Ten-fold cross-validation for receiver operating characteristic (a) and precision-recall curves (b) showing performance of compromised lung volume as a predictor of oxygenation therapy and of intubation (c and $\mathbf{d})$, based on quantitative analysis of chest CT at hospital admittance

identified: $6 \% \mathrm{CL}$ at high sensitivity (90\%; specificity, 43\%); $13 \% \mathrm{CL}$ at high specificity (91\%; sensitivity, 53\%, Fig. 3).

For the intubation outcome, the \%CL model reached CVAUROC 0.86 (weighted fl-score, 0.79; SD, 0.07). Cut-points were $10 \%$ CL at high sensitivity (90\%; specificity, 56\%), and $23 \%$ CL at high specificity (91\%; sensitivity 69\% Fig. 4).

\section{Compromised lung volume and survival}

A moderate negative correlation between \%CL and $\mathrm{PaO}_{2} /$ $\mathrm{FiO}_{2}$ ratio, $r(104)=-0.39, p<0.001$ was observed, highlighting how pulmonary function worsened as \%CL increased.

As a result of multivariate survival analysis, \%CL was predictive of in-hospital mortality (hazard ratio [HR], 1.02;
$95 \% \mathrm{CI}, 1.02$ to $1.05, p=0.01)$, together with age (HR, 1.06; 95\% CI, 1.03-1.10, $p<0.0001$ ), cancer (HR, 3.27; 95\% CI, 1.54-6.94), CRP (HR, 1.03, 95\% CI 1.01-1.05) and CKD (HR, 5.59; 95\% CI, 1.97-15.86). Detailed results are reported in Table 1.

All other variables proved non-significant at univariate analyses and were excluded from the final model.

\section{Discussion}

COVID-19 is a new disease outbreak reaching a pandemic level and a threat to global health [22]. Hospital overcrowding, shortness of ICU beds, oxygen and ventilators have been 
Table 2 Patients' characteristics

\begin{tabular}{ll}
\hline Characteristic & Median (IQR) \\
\hline Age & 66.4 years $(53.8-75.8)$ \\
$\mathrm{BMI}$ & $27.2(24.0-30.1)$ \\
Symptoms onset & 7 days $(3-8.5)$ \\
Body temperature & $37.7^{\circ} \mathrm{C}(36.9-38.4)$ \\
$\mathrm{pO}_{2}$ & $65 \mathrm{mmHg}(53.5-76)$ \\
$\mathrm{pCO}_{2}$ & $34 \mathrm{mmHg}(30.5-38)$ \\
$\mathrm{CRP}$ & $8.1 \mathrm{mg} / \mathrm{L}(2.7-17.9)$ \\
& Total $(N=222)$ \\
Male sex & $163(73 \%)$ \\
Smoke habit & $25(11.2 \%)$ \\
Cancer & $18(8.1 \%)$ \\
Lung disease & $22(9.9 \%)$ \\
Heart Disease & $27(12.16 \%)$ \\
Diabetes & $38(17.1 \%)$ \\
CKD & $10(4.5 \%)$ \\
Medical therapy & \\
Lopinavir-ritonavir & $122(54.9 \%)$ \\
Darunavir plus cobicistat & $80(36.0 \%)$ \\
Hydroxychloroquine & $203(91.4 \%)$ \\
\hline
\end{tabular}

$C R P=\mathrm{C}$-reactive protein $C K D=$ chronic kidney disease

a large-scale concern in in Lombardy (Italy) [13]; thus, accurate and rapid triaging is key to avoid a crisis of the health care network.

Chest x-ray has been proposed as a low-cost tool for detecting lung impairment in patients with suspected COVID-19 [23]. However, its low sensitivity [24] makes it more appropriate for follow-up rather than early diagnosis [25]. Chest CT is instead pivotal to the early diagnosis of COVID-19 due to the ability to detect all disease characteristics [26], and its use has been recommended by the European Society of Radiology in selected patients [27], using a dedicated scanner whenever possible [28]. However, its value is currently limited to visual findings [25], whereas QCT proved to be the tool that allowed for considerable advancements in understanding the ARDS pathophysiology and establishing adequate oxygenation support [29]. These observations led us to perform QCT analysis in all chest CT scans performed at admission on confirmed COVID-19 cases. We identified the volume of compromised lung included in the $-500,100 \mathrm{HU}$ interval as a predictor of oxygenation support and invasive ventilation; this was also significantly correlated with pulmonary dysfunction as measured by the $\mathrm{PaO}_{2} / \mathrm{FiO}_{2}$ ratio and represented a risk factor for in-hospital mortality.

Typical CT appearance of COVID-19 was well described by Chung et al in February 2020 [7] and has been confirmed by several reports $[6,7]$. Based on these findings, Yuan et al have proposed a scoring method to screen patients based on the admittance CT scan [10]. More recently, Li et al also described a visual, quantitative analysis of lung damage, based on a "total severity score" to the degrees of parenchymal loss, correlated with a score of clinical severity [30]. However, these visual characterisations are subjective and unsuited to a systematic disease evaluation and data sharing. Conversely, we reported on a QCT method based on 3D Slicer, which is fast and standardised, and ensures a consistently repeatable evaluation of parenchymal impairment. Some advantages are that (a) it is a free, open-source software untied to any workstation; (b) it has a low learning curve [16]; (c) documentation

Table 3 Details of oxygenation support and results of quantitative lung CT analysis

\begin{tabular}{|c|c|c|c|c|c|}
\hline \multicolumn{6}{|l|}{ Oxygen therapy } \\
\hline & No oxygenation support & Low-flow $\mathrm{O}_{2}$ & High-flow $\mathrm{O}_{2}$ & Intubated & Overall \\
\hline Patients & $56(25 \%)$ & $63(29 \%)$ & $58(26 \%)$ & $45(20 \%)$ & 222 \\
\hline $\mathrm{PaO}_{2} / \mathrm{FIO}_{2}$ &.. & $\begin{array}{l}244.4 \\
(207.4-320)\end{array}$ & $\begin{array}{l}171.43 \\
(122.22-229.63)\end{array}$ & $\begin{array}{l}128.6 \\
(95.4-211.1)\end{array}$ & $\begin{array}{l}192.0 \\
(122.22-251.5)\end{array}$ \\
\hline Death rate & $2(1 \%)$ & $15(7 \%)$ & $26(12 \%)$ & $21(9 \%)$ & $64(29 \%)$ \\
\hline Healing rate & $54(24 \%)$ & $46(21 \%)$ & $30(13 \%)$ & $20(9 \%)$ & $150(68 \%)$ \\
\hline \multicolumn{6}{|c|}{$\begin{array}{l}\text { Lung segmentation } \\
\text { median lung volume \% (IQR) }\end{array}$} \\
\hline & No oxygenation support & Low-flow $\mathrm{O}_{2}$ & High-flow $\mathrm{O}_{2}$ & Intubated & Overall \\
\hline Hyperinflated & $15(4.5-22)$ & $8(2-18)$ & $8(3-12)$ & $3(1-7)$ & $6(2-16)$ \\
\hline Normal & $78.5(71-84)$ & $77(72-83)$ & $77(70-83)$ & $61(45-72)$ & $76(67-83)$ \\
\hline Poorly aerated & $5(4-6)$ & $8(5-13)$ & $11(6-15)$ & $22(13-33)$ & $9(5-15)$ \\
\hline Non-aerated & $1(1-2)$ & $2(1-4)$ & $2(1-3)$ & $6(3-16)$ & $2(1-4)$ \\
\hline Compromised & $6(5-9)$ & $11(7-16)$ & $13.5(7-17)$ & $32(15-50)$ & $12(7-20)$ \\
\hline Total $\left(\mathrm{cm}^{3}\right)$ & $\begin{array}{l}4726.3 \\
(3835.8-5590.9)\end{array}$ & $\begin{array}{l}4115.22 \\
(3246.3-4915.2)\end{array}$ & $\begin{array}{l}3946.51 \\
(2826.1-4663.7)\end{array}$ & $\begin{array}{l}3332.7 \\
(2458.1-4222.6)\end{array}$ & $\begin{array}{l}4057.4 \\
(3132.3-4916.1)\end{array}$ \\
\hline \multicolumn{5}{|l|}{ Predictive value } & Intubation \\
\hline \multicolumn{2}{|c|}{ Poorly aerated [median (IQR)] } & $9(6-13)$ & $8(5-13)$ & $11(7-15)$ & $22(13-33)$ \\
\hline \multicolumn{2}{|l|}{$\mathrm{p}$ value } & $<0.001$ & 0.08 & 0.4 & $<0.001$ \\
\hline \multicolumn{2}{|c|}{ Sensitivity, specificity } & $90.0 \%, 51.1 \%$ & .. & .. & $52.8 \%, 97.1 \%$ \\
\hline \multicolumn{2}{|l|}{ Accuracy } & $80.0 \%$ & .. & .. & $88 \%$ \\
\hline \multicolumn{2}{|c|}{ Compromised lung [median (IQR)] } & $14.5(9-25)$ & $11(7-16)$ & $14.5(10-19)$ & $32(15-50)$ \\
\hline \multicolumn{2}{|c|}{$p$ value } & $<0.001$ & 0.13 & 0.14 & $<0.001$ \\
\hline \multicolumn{2}{|c|}{ Sensitivity, specificity } & $90.0 \%, 51.1 \%$ &.. &.. & $55.6 \%, 97.8 \%$ \\
\hline \multicolumn{2}{|l|}{ Accuracy } & $80.0 \%$ & .. & .. & $89.1 \%$ \\
\hline
\end{tabular}




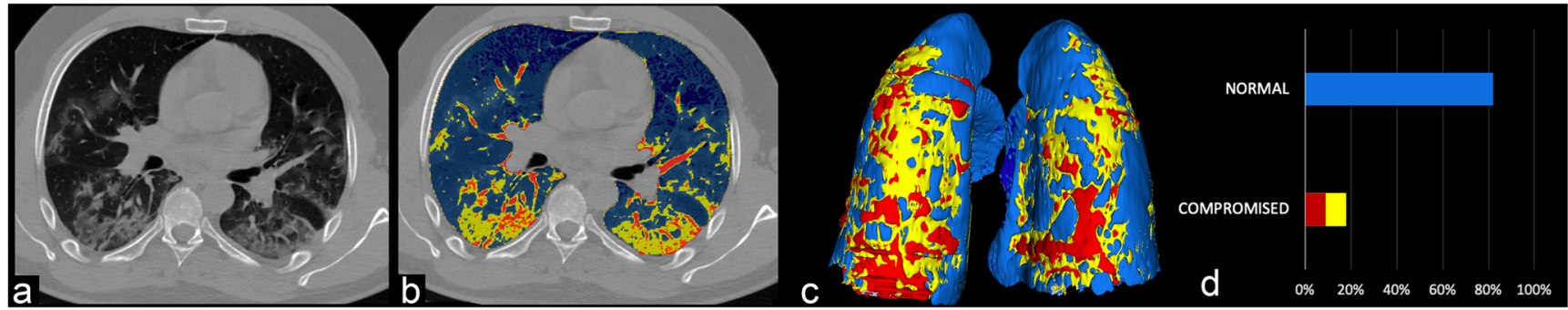

Fig. 3 Quantitative lung CT analysis of a 35-year-old male patient affected by COVID-19. a Non-contrast chest CT at admission showing bilateral ground-glass opacities, interstitial thickening and consolidation in the posterior lung zones. b Semi-automated segmentation using 3D Slicer. Blue areas are normal lung parenchyma; yellow areas represent poorly aerated lung in the $-500,-100 \mathrm{HU}$ interval; red areas represent non- aerated lung and interstitium, in the $-100,100 \mathrm{HU}$ interval. c 3D volumetric representation of both lungs showing multiple red areas in keeping with moderate lung impairment. d Comparison between normal and compromised lung volumes. This patient had $18 \%$ of compromised lung volume, required high-flow oxygenation support through a Venturi Mask. He was discharged after 17 days of sub-intensive care and Internet support are easily found; and (d) it allows for rapid deployment in the ever-changing epicentres of the Coronavirus pandemic.

Colombi et al [31] have used a similar approach to predict the outcomes of COVID-19. They reported good performance of the well-aerated volume (\%WAL, -950 , $-700 \mathrm{HU})$ in predicting the combined outcome of ICU admission and death. Such an outcome, however, would not be informative in our cohort. In fact, 21/48 (66\%) of our deaths happened outside the ICU, and 23/48 (48\%) of patients successfully healed after ICU admittance, remarking that ICU admission should not be generalised as a "worse" outcome. Moreover, they reported the negative predictive value of a BMI surrogate, the adipose tissue measured at the T7-T8 level, whereas in our study BMI was not predictive of the need for oxygenation support or intubation.

Compromised lung was significantly correlated with the $\mathrm{PaO}_{2} / \mathrm{FiO}_{2}$ ratio and increasing pulmonary dysfunction. One reason may be that hypoxemia refractory to oxygenation support is mainly due to intrapulmonary shunt [32] that happens in poorly and non-aerated lung areas, which are well quantified in the \%CL. On the contrary, aerated lung volumes such as \%NAL or $\%$ WAL represent an indirect quantification of this phenomenon and may be more prone to variation during respiratory movements, or ventilation; this may also explain the poor performance of $\% \mathrm{NAL}$ model in our analysis.

Another approach to quantitative analysis has been proposed by Huang et al [33], who have successfully deployed a commercial deep learning algorithm to quantify lung impairment. However, the prediction of oxygenation support was not among their reported outcomes. Despite the promising power of such an approach, the same authors also report that in $8.7 \%$ of cases, the algorithm was unable to correctly identify the lung borders without the help of the radiologist. This need is also shared by our semi-automated approach.

Regardless of the technique used, QCT is seen as the ideal tool that could be able to predict which patient will need a ventilator soon or who no longer requires one. This might help especially in situations with limited hospital resources; in fact, in the process of triaging, the highest priority is to be accorded to those patients whose prognosis is good with intensive care, but poor without it [34]. QCT, particularly the compromised lung volume, may

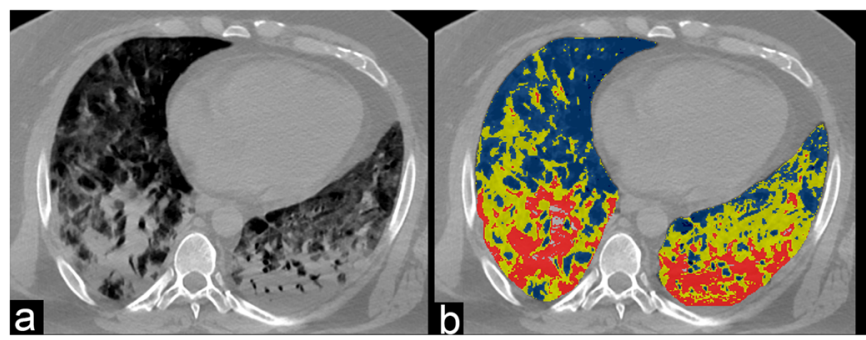

Fig. 4 Quantitative Lung CT analysis of a 43 years old male patient affected by COVID-19. a) Non-contrast chest CT showing extensive areas of bilateral lung consolidation, multiple ground-glass opacities and interstitial thickening and consolidation b) Semi-automated segmentation using 3D Slicer. Blue areas are normal lung parenchyma; yellow areas represent poorly aerated lung in the $-500,-100 \mathrm{HU}$ interval; red areas represent non-aerated lung and interstitium, in the $-100,100 \mathrm{HU}$

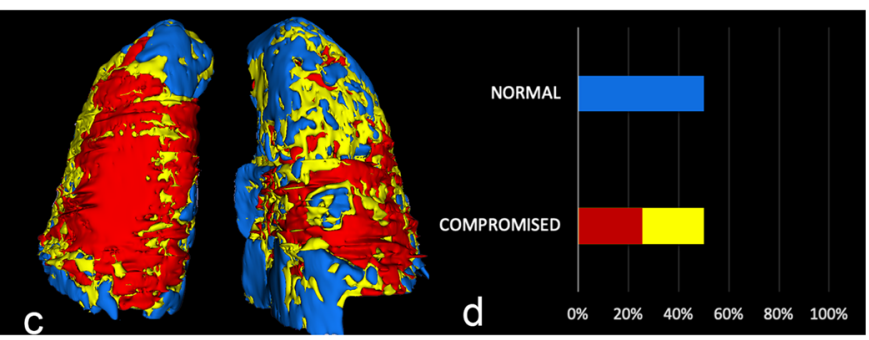

interval. c 3D volumetric representation of both lungs showing extensive red areas of consolidation in keeping with severe pneumonia. d Comparison between normal and compromised lung volumes. This patient had $50 \%$ of compromised lung volume and required immediate intubation and mechanical ventilation. He died after 13 days of intensive care, due to multi-organ failure 
hence be used as a new decision tool, considering its strong correlation not only with respiratory needs but also with in-hospital survival. The need for new metrics specific to COVID-19 is remarked by the low performance of a validated score for pneumonia (CURB-65) that was tested for comparison; most patients (58\%) were graded as low-mortality risk (group 1); still, we observed a $15 \%$ death rate, much higher than the presumed $1.5 \%$ [15].

This study has several limitations. Firstly, it has a retrospective design. Secondly, we did not perform a repeat CT on the healed patients before discharge, to check whether the physiological lung volumes had been restored; this could be the subject of a future study.

Regarding the segmentation process, we included the interstitial structures in the analysed volume (e.g. segmental arteries and bronchi). By partially falling in the same threshold as the non-aerated lung, these may have reduced precision. However, the event might have been irrelevant to the outcome. Firstly, because the poorly aerated volume alone was a strong outcome predictor; secondly, because the same method was applied to all patients thus counterbalancing the potential inaccuracy; thirdly, the added volume of the lung interstitium may be considered negligible compared to the compromised lung volume in the setting of severe pneumonia. On the contrary, this choice allowed for a fast segmentation process.

Regarding respiratory support, $20 \%$ of our patients required invasive mechanical ventilation, a higher number compared to $2.5 \%$ reported by $\mathrm{Wu}$ et al and $17 \%$ by Wang and Zhou et al These observations may be partially due to the higher age of the Italian population [35]. Indeed, the median age of our cohort (66 years) was above the ones previously reported in China by Wu et al [36] (51 years), Wang et al [37] and Fei Zhou et al [4] (56 years).

The main strengths of our study are the high number of patients enrolled, the completeness of clinical data, the high statistical significance of all tests conducted and the use of a rapidly reproducible and scalable method for QCT.

In conclusion, lung QCT provides new metrics of COVID19 and has a promising role in predicting its clinical outcome. The percentage of lung parenchyma in the $-500,100 \mathrm{HU}$ interval, namely the compromised lung volume (\%CL), has shown high accuracy in predicting the need for oxygenation support and mechanical ventilation and is a risk factor for inhospital death.

We empirically identified different cut points of compromised lung volume: patients presenting with \%CL values in the $6-23 \%$ range are at risk for needing oxygenation therapy; values above $23 \%$ are at risk for intubation. These results strengthen the evidence of QCT as an ideal tool for easing the triaging process of COVID-19.
Acknowledgements The authors acknowledge Dr. Mrakic, Dr. Giannitto, Prof. Cecconi and Prof. Protti for their efforts in the research in the field of the COVID-19 pandemic.

Funding information The authors state that this work has not received any funding.

\section{Compliance with ethical standards}

Guarantor The scientific guarantor of this publication is Ezio Lanza.

Conflict of interest The authors of this manuscript declare no relationships with any companies whose products or services may be related to the subject matter of the article.

Statistics and biometry Ezio Lanza, Pierandrea Morandini, Giovanni Angelotti and Viktor Savevski have significant statistical expertise.

Informed consent Written informed consent was obtained from all subjects (patients) in this study.

Ethical approval Institutional Review Board approval was obtained.

Methodology
- retrospective
- observational
- performed at one institution

\section{References}

1. Guan W-J, Ni Z-Y, Hu Y et al (2020) Clinical characteristics of coronavirus disease 2019 in China. N Engl J Med. https://doi.org/ 10.1056/NEJMoa2002032

2. Situation update for the EU/EEA and the UK, as of 27 March 2020. https://www.ecdc.europa.eu/en/cases-2019-ncov-eueea. Accessed 28 Mar 2020

3. McMichael TM, Currie DW, Clark S et al (2020) Epidemiology of Covid-19 in a long-term care facility in King County, Washington. N Engl J Med. https://doi.org/10.1056/NEJMoa2005412

4. Zhou F, Yu T, Du R et al (2020) Clinical course and risk factors for mortality of adult inpatients with COVID-19 in Wuhan, China: a retrospective cohort study. Lancet 395:1054-1062

5. Poston JT, Patel BK, Davis AM (2020) Management of critically ill adults with COVID-19. JAMA. https://doi.org/10.1001/jama.2020. 4914

6. Shi H, Han X, Jiang N et al (2020) Radiological findings from 81 patients with COVID-19 pneumonia in Wuhan, China: a descriptive study. Lancet Infect Dis 20:425-434

7. Chung M, Bernheim A, Mei X et al (2020) CT imaging features of 2019 novel coronavirus (2019-nCoV). Radiology 295:202-207

8. Li Y, Xia L (2020) Coronavirus disease 2019 (COVID-19): role of chest $\mathrm{CT}$ in diagnosis and management. AJR Am J Roentgenol:1-7

9. Lescure F-X, Bouadma L, Nguyen D et al (2020) Clinical and virological data of the first cases of COVID-19 in Europe: a case series. Lancet Infect Dis. https://doi.org/10.1016/S1473-3099(20) 30200-0

10. Yuan M, Yin W, Tao Z, Tan W, Hu Y (2020) Association of radiologic findings with mortality of patients infected with 2019 novel coronavirus in Wuhan, China. PLoS One 15:e0230548

11. Nishiyama A, Kawata N, Yokota $\mathrm{H}$ et al (2020) A predictive factor for patients with acute respiratory distress syndrome: CT lung 
volumetry of the well-aerated region as an automated method. Eur J Radiol 122:108748

12. Ichikado K, Suga M, Muranaka H et al (2006) Prediction of prognosis for acute respiratory distress syndrome with thin-section CT: validation in 44 cases. Radiology 238:321-329

13. Nacoti M, Ciocca A, Brambillasca P, et al (2020) At the epicenter of the Covid-19 pandemic and humanitarian crises in Italy: changing perspectives on preparation and mitigation | catalyst non-issue content. NEJM Catalyst Innovations in Care Delivery

14. Zhu N, Zhang D, Wang W et al (2020) A novel coronavirus from patients with pneumonia in China, 2019. N Engl J Med 382:727733

15. Lim WS, van der Eerden MM, Laing R et al (2003) Defining community acquired pneumonia severity on presentation to hospital: an international derivation and validation study. Thorax 58:377-382

16. Fedorov A, Beichel R, Kalpathy-Cramer J et al (2012) 3D slicer as an image computing platform for the quantitative imaging network. Magn Reson Imaging 30:1323-1341

17. Yip SSF, Parmar C, Blezek D et al (2017) Application of the 3D slicer chest imaging platform segmentation algorithm for large lung nodule delineation. PLoS One 12:e178944

18. Sharkh HA, Makhoul N (2020) In-house surgeon-led virtual surgical planning for maxillofacial reconstruction. J Oral Maxillofac Surg 78(4):651-660. https://doi.org/10.1016/j.joms.2019.11.013

19. Cressoni M, Gallazzi E, Chiurazzi C et al (2013) Limits of normality of quantitative thoracic CT analysis. Crit Care 17:R93

20. Blagus R, Lusa L (2013) SMOTE for high-dimensional class-imbalanced data. BMC Bioinformatics 14:106

21. Luque-Fernandez MA, Redondo-Sánchez D, Maringe C (2019) cvauroc: command to compute cross-validated area under the curve for ROC analysis after predictive modeling for binary outcomes. Stata J 19:615-625

22. Madjid M, Safavi-Naeini P, Solomon SD, Vardeny O (2020) Potential effects of coronaviruses on the cardiovascular system: a review. JAMA Cardiol. https://doi.org/10.1001/jamacardio.2020. 1286

23. Bandirali M, Sconfienza LM, Serra R, et al (2020) Chest X-ray findings in asymptomatic and minimally symptomatic quarantined patients in Codogno, Italy. Radiology 201102

24. Wong HYF, Lam HYS, Fong AH-T et al (2019) Frequency and distribution of chest radiographic findings in COVID-19 positive patients. Radiology 201160

25. Ng M-Y, Lee EY, Yang $J$ et al (2020) Imaging profile of the COVID-19 infection: radiologic findings and literature review. Radiology: Cardiothoracic Imaging 2:e200034
26. Ai T, Yang Z, Hou H et al (2020) Correlation of chest CT and RTPCR testing in coronavirus disease 2019 (COVID-19) in China: a report of 1014 cases. Radiology 200642

27. Revel M-P, Parkar AP, Prosch H et al (2020) COVID-19 patients and the radiology department-advice from the European Society of Radiology (ESR)and the European Society of Thoracic Imaging (ESTI). Eur Radiol

28. Politi LS, Balzarini L (2020) The radiology department during the COVID-19 pandemic: a challenging, radical change. Eur Radiol. https://doi.org/10.1007/s00330-020-06871-0

29. Gattinoni L, Cressoni M (2010) Quantitative CT in ARDS: towards a clinical tool? Intensive Care Med 36:1803-1804

30. Li K, Fang Y, Li W et al (2020) CT image visual quantitative evaluation and clinical classification of coronavirus disease (COVID-19). Eur Radiol. https://doi.org/10.1007/s00330-02006817-6

31. Colombi D, Bodini FC, Petrini M, et al (2020) Well-aerated lung on admitting chest CT to predict adverse outcome in COVID-19 pneumonia. Radiology 201433

32. Radermacher P, Maggiore SM, Mercat A (2017) Fifty years of research in ARDS. Gas exchange in acute respiratory distress syndrome. Am J Respir Crit Care Med 196:964-984

33. Huang L, Han R, Ai T et al (2020) Serial quantitative chest CT assessment of COVID-19: deep-learning approach. Radiology: Cardiothoracic Imaging 2:e200075

34. Swiss Academy Of Medical Sciences (2020) COVID-19 pandemic: triage for intensive-care treatment under resource scarcity. Swiss Med Wkly 150:w20229

35. (2014) Elderly population. Organisation for Economic CoOperation and Development (OECD). doi: https://doi.org/10. 1787/8d805ea1-en

36. Wu C, Chen X, Cai Y et al (2020) Risk factors associated with acute respiratory distress syndrome and death in patients with coronavirus disease 2019 pneumonia in Wuhan, China. JAMA Intern Med. https://doi.org/10.1001/jamainternmed.2020.0994

37. Wang $\mathrm{D}, \mathrm{Hu} \mathrm{B}, \mathrm{Hu} \mathrm{C}$ et al (2020) Clinical characteristics of 138 hospitalized patients with 2019 novel coronavirus-infected pneumonia in Wuhan, China. JAMA. https://doi.org/10.1001/jama. 2020.1585

Publisher's note Springer Nature remains neutral with regard to jurisdictional claims in published maps and institutional affiliations. 